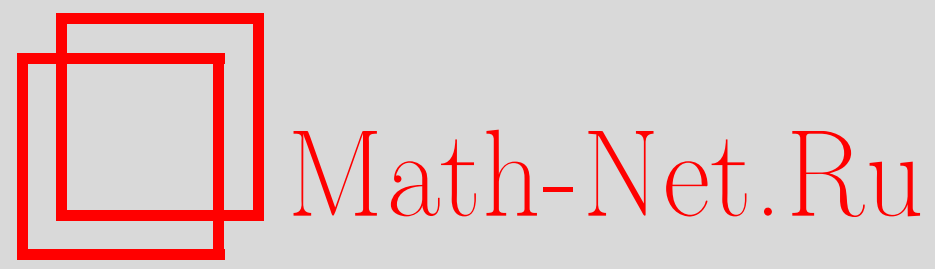

Д. Н. Туляков, О некоторой процедуре нахождения асимптотических разложений для решений разностных уравнений, Совр. пробл. матем., 2007, выпуск $9,45-53$

DOI: https://doi.org/10.4213/spm16

Использование Общероссийского математического портала MathNet.Ru подразумевает, что вы прочитали и согласны с пользовательским соглашением

http://www . mathnet.ru/rus/agreement

Параметры загрузки:

IP: 44.207 .124 .84

26 апреля 2023 г., 18:27:45

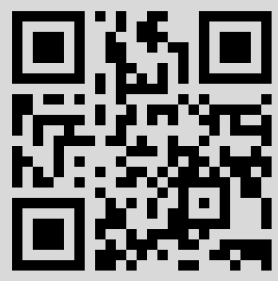




\title{
О некоторой процедуре нахождения асимптотических разложений для решений разностных уравнений ${ }^{1}$
}

\author{
Д. Н. Туляков
}

\section{1. Введение}

Из общей теории разностных уравнений известно, что у линейного рекуррентного соотношения с полиномиальными по индексу коэффициентами существует базис решений, имеющих асимптотическое разложение по $n$ специального вида. В работах [1]-[3] доказана теорема существования для разностных уравнений с полиномиальными по аргументу коэффициентами. Её точная формулировка такова:

Теорема сУЩествования (G.D. Birkhoff, W. J. Trjitzinsky, 1932). Любое линейное разностное уравнение $n$-го порядка

$$
\sum_{i=0}^{n} a_{i}(z) q(z+i)=0 \quad\left(a_{0} \not \equiv 0, \quad a_{n} \not \equiv 0\right)
$$

с полиномиальными коэффициентами $a_{i}$ имеет ровно $n$ линейно независимых формальных решений следующего общего вида:

$$
\begin{gathered}
q(z)=e^{Q(z)} z^{r} \sum_{s=0}^{\infty} z^{-\frac{s}{p}} \sum_{k=0}^{m} C_{s, k} \ln ^{k} z, \quad Q(z)=\mu z \ln z+\sum_{i=1}^{p} \nu_{i} z^{\frac{i}{p}}, \\
p \in \mathbb{N}, \quad \mu p \in \mathbb{Z}, \quad m \in \mathbb{N} \cup\{0\} .
\end{gathered}
$$

Если плоскость комплексного переменного z разбить на области кривыми $\operatorname{Re}\left(Q_{i}(z)\right)=\operatorname{Re}\left(Q_{j}(z)\right), i, j=1, \ldots, n$, то в каждой такой области, уходящей в бесконечность, существуют $n$ линейно независимых аналитических решений уравнения (1), имеющих найденную асимптотику.

\footnotetext{
${ }^{1}$ Работа частично поддержана грантом научных школ НШ-1551.2003.1, программой № 1 ОМН РАН, исследовательским грантом РФФИ-05-01-00697 и грантом ИНТАС 03-51-6637.
}

(C) Туляков Д.Н., 2007 
C помощью уравнения любое решение уравнения (1) можно продолжить по вещественному направлению, но при переходе в другую область асимптотика, вообще говоря, меняется.

Общая процедура нахождения таких разложений достаточно сложна, но в большинстве случаев хватает упрощённой процедуры, применение которой будет изложено на примере соотношения (3):

$$
\begin{aligned}
& (n+1)(16 n-15) q_{n+1}=\left(128 n^{3}+40 n^{2}-82 n-45\right) q_{n} \\
& \quad-n\left(256 n^{3}-240 n^{2}+64 n-7\right) q_{n-1}+(16 n+1) n(n-1) q_{n-2} .
\end{aligned}
$$

Итак, нашей целью будет доказательство следующей теоремы о поведении решений соотношения (3).

Теорема. Существует базис из трёх решений разностного уравнения (3) со следующими асимптотиками:

$$
\begin{aligned}
& q_{1}(n)=\left(\frac{4 n}{e}\right)^{n} \frac{e^{\sqrt{2 n}}}{\sqrt[4]{n}}\left(1+\frac{97}{96 \sqrt{2 n}}-\frac{2207}{36864 n}\right. \\
&\left.-\frac{13308187}{53084160 n \sqrt{2 n}}+O\left(n^{-2}\right)\right), \\
& q_{2}(n)=\left(\frac{4 n}{e}\right)^{n} \frac{e^{-\sqrt{2 n}}}{\sqrt[4]{n}}\left(1-\frac{97}{96 \sqrt{2 n}}-\frac{2207}{36864 n}\right. \\
&\left.+\frac{13308187}{53084160 n \sqrt{2 n}}+O\left(n^{-2}\right)\right), \\
& q_{3}(n)=\left(\frac{e}{16 n}\right)^{n} \frac{1}{n \sqrt{n}}\left(1-\frac{77}{96 n}+\frac{9745}{18432 n^{2}}\right. \\
&\left.-\frac{7543057}{26542080 n^{3}}+O\left(n^{-4}\right)\right) .
\end{aligned}
$$

Асимптотические ряды эффективно продолжаются до членов любого заданного порядка.

\section{2. Доказательство теоремы}

Обозначим $d(z)=q(z+1) / q(z)$. При подстановке в (1) получим соотношение

$$
\sum_{i=0}^{n} a_{i}(z) \prod_{k=1}^{i} d(z+k-1)=0,
$$


которое для нашего случая (3) имеет вид

$$
\begin{aligned}
\left(16 z^{2}\right. & +65 z+51) d(z+2) d(z+1) d(z) \\
& -\left(128 z^{3}+808 z^{2}+1614 z+975\right) d(z+1) d(z) \\
& +\left(256 z^{4}+1808 z^{3}+4768 z^{2}+5561 z+2418\right) d(z) \\
& -\left(16 z^{3}+81 z^{2}+131 z+66\right)=0 .
\end{aligned}
$$

Теперь находим асимптотику $d(z)$ в несколько этапов.

$1^{\circ}$. Главный член асимптотики $d(z)$ находится из рассмотрения диаграммы Ньютона характеристического уравнения, поскольку он одинаков для $d(z), d(z+1)$ и так далее. Возьмём множество точек $\mathbb{R}^{2}\left\{\left(i, \operatorname{deg}\left(a_{i}\right)\right) \mid i=1, \ldots, n\right\}$, построим выпуклую оболочку этого множества (это многоугольник) и обозначим через $S$ набор тех его сторон, относительно которых многоугольник находится "снизу". Формально это значит, что $y \leqslant b x+c-$ уравнение полуплоскости, содержащей многоугольник, а её граница прямая, содержащая сторону.

Пусть $\left(m_{j}, b_{j}\right)$ - соответственно длина горизонтальной проекции $j$-й стороны и её угловой коэффициент, а через $x_{j}$ обозначим абсциссы вершин верхней части многоугольника Ньютона (концов сторон из набора $S$ ). Тогда

$$
\begin{gathered}
x_{1}=0, \quad m_{j}=x_{j+1}-x_{j}>0, \\
b_{j}=\frac{\operatorname{deg}\left(a_{x_{j+1}}\right)-\operatorname{deg}\left(a_{x_{j}}\right)}{x_{j+1}-x_{j}}, \quad b_{j+1}<b_{j} .
\end{gathered}
$$

Обозначим

$$
P_{j}(t)=\sum_{k=0}^{m_{j}} d_{k}^{(j)} t^{k},
$$

где

$$
d_{k}^{(j)}=\operatorname{Coeff}\left(a_{x_{j}+k}, \operatorname{deg}\left(a_{x_{j}}\right)+k b_{j}\right) .
$$

Если $\lambda_{k}^{(j)}-$ корни полиномов $P_{j}(t)$, и $\nu_{k}^{(j)}-$ соответствующие им кратности, то есть ровно $\nu_{k}^{(j)}$ асимптотических решений $d(z)$ с главным членом $\lambda_{k}^{(j)} z^{-b_{j}}$. Для решений, у которых $\nu=1$, можно сразу переходить к 3 этапу. В других случаях решения надо разделить на этапе 2. Предложенным способом решения разделяются не всегда, в частности, решение вида (2) с $m \neq 0$ не отделяется, но для уравнения (3) он применим. 
Применив эти построения для (5a), последовательно получим: множество $\{(3,2),(2,3),(1,4),(0,3)\}$; двухзвенную ломаную в качестве верхней части многоугольника Ньютона, и остальные величины:

$$
\begin{gathered}
x_{1}=0, \quad x_{2}=1, \quad x_{3}=3, \quad m_{1}=1, \quad m_{2}=2, \quad b_{1}=1, \quad b_{2}=-1, \\
P_{1}(t)=256 t-16, \quad P_{2}(t)=16 t^{2}-128 t+256, \\
\nu_{1}^{(1)}=1, \quad \lambda_{1}^{(1)}=1 / 16, \quad \nu_{1}^{(2)}=2, \quad \lambda_{1}^{(2)}=4 .
\end{gathered}
$$

Поэтому у уравнения (5а) есть одно решение $d(z) \approx(16 z)^{-1}$ и два решения $d(z) \approx 4 z$.

$2^{\circ}$. Уточнение асимптотики и разделение решений. Выберем фиксированный главный член $\lambda_{k}^{(j)} z^{-b_{j}}$ из найденных на этапе 1. Сделаем подстановку $d(z)=\lambda_{k}^{(j)} z^{-b_{j}} \tilde{d}(z)$ в (5), и будем искать решения $\tilde{d}(z)$ с главным членом 1 . Для этого сгруппируем левую часть специальным образом:

$$
\sum_{i=0}^{n}\left(\tilde{a}_{i}(z) \sum_{s=0}^{i}(-1)^{i-s}\left(\begin{array}{l}
i \\
s
\end{array}\right) \prod_{k=1}^{s} \tilde{d}(z+k-1)\right) .
$$

Такая группировка соответствует разложению характеристического полинома от $\tilde{d}$ по степеням $(\tilde{d}-1)$. Коэффициенты $\tilde{a}_{i}(z)$ определяются единственным образом и являются алгебраическими функциями. Из определения величин $b_{j}, \lambda_{k}^{(j)}$ и $\nu=\nu_{k}^{(j)}$ следует, что наибольший порядок роста по $z$ на бесконечности среди $\tilde{a}_{i}(z)$ будет для $i=\nu$ (возможно и для некоторых других $\left.i\right)$, а для всех $i<\nu$ порядок роста $\tilde{a}_{i}(z)$ по $z$ будет строго меньше. Считаем, что $\nu>1$.

Вновь строим диаграмму: точки $\left(i ; \operatorname{ord}\left(\tilde{a}_{i}\right)\right)$ для $i=0, \ldots, \nu$ берём в качестве начального множества. Здесь ord понимается как порядок роста и обобщает deg: если при больших $|z| f(z)=O\left(z^{\alpha}\right)$, то $\operatorname{ord}(f)=\alpha$. Проводим опорную прямую к последней точке, и пусть её угловой коэффициент $\widetilde{m}<1$. Строим полином $\widetilde{P}(t)=\sum_{k=0}^{\nu} \operatorname{Coeff}\left(\tilde{a}_{k}, \operatorname{ord}\left(\tilde{a}_{\nu}\right)+\widetilde{m}(k-\nu)\right) t^{k}$. Каждый его коэффициент - или коэффициент при главном члене асимптотики, или 0. Тогда каждый простой корень $\tau$ полинома $\widetilde{P}(t)$ соответствует отделившемуся решению $\tilde{d}(z) \approx 1+\tau z^{-\widetilde{m}}$. Снова приводим выкладки для интересующей нас конкретной ситуации. На этапе 1 мы убедились, что есть только один случай $\nu \neq 1$. Производим подстановку $d(z)=4 z \tilde{d}(z)$ в (5а) и группируем нужным 
образом:

$$
\begin{aligned}
-\left(1024 n^{5}+7232 n^{4}+17792 n^{3}+18112 n^{2}+6528 n\right) \\
\quad \times[\tilde{d}(z+2) \tilde{d}(z+1) \tilde{d}(z)-3 \tilde{d}(z+1) \tilde{d}(z)+3 \tilde{d}(z)-1] \\
\quad-\left(1024 n^{5}+6720 n^{4}+14624 n^{3}+12912 n^{2}+3984 n\right) \\
\quad \times[\tilde{d}(z+1) \tilde{d}(z)-2 \tilde{d}(z)+1] \\
\quad+\left(1024 n^{4}+5056 n^{3}+6268 n^{2}+1944 n\right)[\tilde{d}(z)-1] \\
\quad+\left(512 n^{4}+1904 n^{3}+1149 n^{2}-469 n+66\right)=0 .
\end{aligned}
$$

Точки $(0 ; 4),(1 ; 4),(2 ; 5)$. Угловой коэффициент $\widetilde{m}=1 / 2<1$. Корни полинома $\widetilde{P}(t)=-1024 t^{2}+512$ простые и равны $\pm \sqrt{1 / 2}$. Получаем асимптотику $d(z) \approx 4 z(1 \pm \sqrt{1 /(2 z)})$.

$3^{\circ}$. Продолжение асимптотического ряда методом итераций. Используем начало асимптотики отделившегося решения, сделаем подстановку $d(z)=\lambda_{k}^{(j)} z^{-b_{j}}\left(1+\tau z^{-\tilde{m}}\right) \tilde{d}(z)$ и повторим преобразование (5) к виду (6). Если этап 2 был пропущен, то $\tau$ отсутствует. Поскольку решение отделившееся, то слагаемое $\tilde{a}_{1}(z)(\tilde{d}(z)-1)$, соответствующее $i=1$, является ведущим. Переносим все остальные слагаемые в правую часть, выражаем $\tilde{d}(z)$ из левой части и в результате получаем итерационное соотношение

$$
\tilde{d}(z):=1-\frac{1}{\tilde{a}_{1}(z)} \sum_{\substack{i=0 \\
i \neq 1}}^{n}\left(\tilde{a}_{i}(z) \sum_{s=0}^{i}(-1)^{i-s}\left(\begin{array}{l}
i \\
s
\end{array}\right) \prod_{k=1}^{s} \tilde{d}(z+k-1)\right) .
$$

Стартуя с функции $\tilde{d}(z)=1$, из соотношения (7) будем получать функции, асимптотическое разложение которых (с ростом числа итераций) стабилизируется в силу лемм 2 и 3 из приложения.

$4^{\circ}$. Осталось возвратиться к $d(z)$ и по асимптотическому ряду $d(z)$ получить асимптотический ряд $q(z)$. Это требует суммирования асимптотических рядов и не представляет трудностей:

$$
q(z)=\mathrm{const} \prod_{t}^{z-1} d(t)=\mathrm{const} \cdot \exp \left(\sum_{t}^{z-1} \ln d(t)\right) .
$$

Итак, для соотношения (5а) делаем преобразование к виду (6) после каждой из следующих подстановок:

$$
\begin{gathered}
d(z)=(4 z+2 \sqrt{2 z}) \tilde{d}(z), \quad d(z)=(4 z-2 \sqrt{2 z}) \tilde{d}(z), \\
d(z)=\tilde{d}(z) /(16 z) .
\end{gathered}
$$


Для краткости и наглядности будем проводить преобразования (5a) сразу для всех случаев, записав подстановки в виде $d(z)=$ $d_{0}(z) \tilde{d}(z)$.

Представим левую часть соотношения (5a) как применение оператора $L$ к функции $d$, то есть

$$
\begin{aligned}
L[d](z)= & a_{0}+a_{1} d(z)+a_{2} d(z) d(z+1)+a_{3} d(z) d(z+1) d(z+2), \\
& a_{0}=-\left(16 z^{3}+81 z^{2}+131 z+66\right), \\
& a_{2}=-\left(128 z^{3}+808 z^{2}+1614 z+975\right), \\
& a_{1}=\left(256 z^{4}+1808 z^{3}+4768 z^{2}+5561 z+2418\right), \\
& a_{3}=16 z^{2}+65 z+51 .
\end{aligned}
$$

Применим подстановку,

$$
\begin{array}{rlrl}
L[d](z) & =A_{0}+A_{1} \tilde{d}(z)+A_{2} \tilde{d}(z) \tilde{d}(z+1)+A_{3} \tilde{d}(z) \tilde{d}(z+1) \tilde{d}(z+2), \\
A_{0} & =a_{0}, & & A_{1}=a_{1} d_{0}(z), \\
A_{2} & =a_{2} d_{0}(z) d_{0}(z+1), & & A_{3}=a_{3} d_{0}(z) d_{0}(z+1) d_{0}(z+2) .
\end{array}
$$

Преобразуем к виду (6),

$$
\begin{aligned}
& L[d](z)=\sum_{i=0}^{3}\left(B_{i} \sum_{s=0}^{i}(-1)^{i-s}\left(\begin{array}{l}
i \\
s
\end{array}\right) \prod_{k=1}^{s} \tilde{d}(z+k-1)\right), \\
& B_{3}=A_{3}, \\
& B_{1}=A_{1}+2 A_{2}+3 A_{3}, \quad B_{0}=A_{0}+A_{1}+A_{2}+A_{3} .
\end{aligned}
$$

Запишем равенство (7) для нашего случая, используя $L[d]$ :

$$
\begin{aligned}
\tilde{d}(z) & =1-\frac{1}{B_{1}} \sum_{\substack{i=0 \\
i \neq 1}}^{3}\left(B_{i} \sum_{s=0}^{i}(-1)^{i-s}\left(\begin{array}{l}
i \\
s
\end{array}\right) \prod_{k=1}^{s} \tilde{d}(z+k-1)\right) \\
& =\tilde{d}(z)-\frac{L[d](z)}{B_{1}} .
\end{aligned}
$$

Возвращаясь к функции $d$, мы видим, что итерационное соотношение можно записать в виде

$$
\begin{aligned}
d(z) & =d(z)-\frac{d_{0}(z) L[d](z)}{B_{1}} \\
& =d(z)-\frac{L[d](z)}{a_{1}+2 a_{2} d_{0}(z+1)+3 a_{3} d_{0}(z+1) d_{0}(z+2)} .
\end{aligned}
$$




\section{3. Приложение}

В данном разделе будут доказаны леммы о асимптотическом поведении некоторых характерных для основного раздела величин (леммы 2 и 3 ), которые доказываются на основе одного технического результата (лемма 1 ).

Лемма 1. Пусть $w(z)$ - аналитическая функция в секторе $\operatorname{Arg}(z) \in[-\alpha ; \alpha], \alpha<\pi$, с ограниченым модулем. Если для граничных значений $w(z)$ выполняется неравенство $|w(z)|<|z|_{+}^{a} \varphi(|z|)$, где $|z|_{+}=\max (1,|z|), a<0, \varphi$ есть функиия медленного роста в $\mathbb{R}_{+}$с условиями: $|t|_{+} \varphi(t)$ не убъвает, $\frac{\varphi(t)}{|t|_{+}}$не возрастает, то для произвольного $\varepsilon>0$ в секторе $|\operatorname{Arg}(z)| \leqslant \alpha-\varepsilon$ $w^{\prime}(z)=O\left(\frac{\varphi(|z|)}{|z|}\right)$.

$\mathrm{C}$ помощью возведения в степень задачи с различными $\alpha$ сводятся друг к другу, поэтому достаточно доказать неравенство для случая $\alpha=\pi / 2$. Для тех $z$ из сектора, у которых $|z| \leqslant 1$, модуль производной ограничен некоторой константой, поэтому считаем, что $|z|>1$. Напишем формулу Коши для производной, взяв контур из отрезка мнимой оси и правой полуокружности, построенной на нём, как на диаметре. Преобразуем и начнём неограниченно расширять контур. Получим

$$
\begin{aligned}
w^{\prime}(z) & =\frac{1}{2 \pi i} \oint_{\gamma} \frac{w(s) d s}{(s-z)^{2}} \\
& =\lim _{R \rightarrow \infty}\left(\frac{1}{2 \pi} \int_{-\pi / 2}^{\pi / 2} \frac{w\left(R e^{i t}\right) R e^{i t} d t}{\left(R e^{i t}-z\right)^{2}}-\frac{1}{2 \pi} \int_{-R}^{R} \frac{w(i t) d t}{(i t-z)^{2}}\right) \\
& =0-\frac{1}{2 \pi} \int_{-\infty}^{\infty} \frac{w(i t) d t}{(i t-z)^{2}}, \\
\left|w^{\prime}(z)\right| & <\frac{1}{2 \pi} \int_{-\infty}^{\infty} \frac{|w(i t)| d t}{(i t-z)(-i t-\bar{z})} .
\end{aligned}
$$

Учитываем условия $|z|>1,|\operatorname{Arg}(z)|<\frac{\pi}{2}-\varepsilon$ и свойства $\varphi$. Получим

$$
\begin{aligned}
\left|w^{\prime}(z)\right| & <\frac{1}{2 \pi} \int_{-\infty}^{\infty} \frac{|t|_{+}^{a} \varphi(|t|) d t}{t^{2}-2 \operatorname{Im}(z) t+|z|^{2}} \\
& =\frac{1}{\pi} \int_{0}^{\infty} \frac{|t|_{+}^{a} \varphi(t) d t \cdot\left(t^{2}+|z|^{2}\right)}{\left(t^{2}+|z|^{2}\right)^{2}-4 \operatorname{Im}^{2}(z) t^{2}}
\end{aligned}
$$




$$
\begin{aligned}
& <\frac{1}{\pi \sin ^{2} \varepsilon} \int_{0}^{\infty} \frac{|t|_{+}^{a} \varphi(t) d t}{t^{2}+|z|^{2}} \\
& <\frac{1}{\pi \sin ^{2} \varepsilon}\left(\int_{0}^{|z|} \frac{|t|_{+}^{a} \varphi(t) d t}{|z|^{2}}+\int_{|z|}^{\infty} \frac{|t|_{+}^{a} \varphi(t) d t}{t^{2}}\right) \\
& <\frac{\varphi(|z|)|z|^{-1}}{\pi \sin ^{2} \varepsilon}\left(\int_{0}^{|z|}|t|_{+}^{a-1} d t+\int_{|z|}^{\infty}|t|_{+}^{a-1} d t\right) \\
& =\frac{1-a}{-a \pi \sin ^{2} \varepsilon} \frac{\varphi(|z|)}{|z|} .
\end{aligned}
$$

При выполнении обратного преобразования изменится только константа.

Лемма 2. Пусть функиия $d(z)$ аналитическая в некотором секторе, содержащем положительное направление, и имеет там представление $d(z)=1+z^{a} \varphi(z)(1+\overline{\bar{o}}(1))$, где $a<0 u$ $\ln |\varphi(z)|=\overline{\bar{o}}(\ln |z|)$ при $|z| \rightarrow \infty$. Рассмотрим величинь

$$
f_{n}(z)=\sum_{s=0}^{n}(-1)^{n-s}\left(\begin{array}{l}
n \\
s
\end{array}\right) \prod_{k=1}^{s} d(z+k-1) .
$$

Тогда для натуральных $n$ в любом более узком секторе верно представление

$$
f_{n}(z)= \begin{cases}z^{n a} \varphi^{n}(z)(1+\overline{\bar{o}}(1)), & \text { если } a>-1, \\ O\left(z^{-n} \varphi(z)|\varphi(z)|_{+}^{n-1}\right), & \text { если } a=-1, \\ O\left(z^{a+1-n} \varphi(z)\right), & \text { если } a<-1 .\end{cases}
$$

Будем использовать обозначение $\Delta$ для оператора разности: $\Delta h(z)=h(z+1)-h(z)$. Поскольку $\Delta h(z)=\int_{0}^{1} h^{\prime}(z+t) d t$, то для оценки разности можно применять лемму 1. Легко установить, что величины удовлетворяют рекуррентному соотношению

$$
f_{n+1}(z)=f_{n}(z+1) d(z)-f_{n}(z)=\Delta f_{n}(z)+f_{n}(z+1)(d(z)-1)
$$

и начальному условию $f_{0}=1$. Следовательно, $f_{1}(z)=d(z)-1$, и база индукции верна. Индуктивный переход легко делается для каждого из трёх случаев сравнения $a \mathrm{c}-1$. На каждом шаге индукции мы сужаем сектор. Поскольку величина сужения может быть выбрана сколь угодно малой, то на фиксированном шаге действительно можно получить любой меньший сектор. 
Лемма 3. Пусть для функиии $d(z)$ выполнены условия предъдущей леммы, при этом $а \in(-1 ; 0)$. Тогда результат леммы 2 дополняется оценкой изменения величин $f_{n}(z)$ при изменении $d(z)$. Пусть $d(z)$ изменяется на $\delta d(z)=z^{b} \psi(z)(1+\overline{\bar{o}}(1))$, где $b<a, \ln |\psi(z)|=\overline{\bar{o}}(\ln |z|)$ при $|z| \rightarrow \infty$. Тогда изменение $\delta f_{n}(z)$ величины $f_{n}(z)$ имеет оценку

$$
\delta f_{n}(z)=n z^{(n-1) a+b} \varphi^{n-1}(z) \psi(z)(1+\overline{\bar{o}}(1)) .
$$

Подставим в (8) $d(z)+\delta d(z)$ вместо $d(z), f_{n}(z)+\delta f_{n}(z)$ вместо $f_{n}(z)$, вычтем исходное соотношение (8) и преобразуем. Получим $\delta f_{n+1}(z)=\Delta \delta f_{n}(z)+f_{n}(z+1) \delta d(z)+\delta f_{n}(z+1)(d(z)+\delta d(z)-1)$.

Используя предыдущую лемму и начальное условие $\delta f_{1}(z)=$ $\delta(d(z)-1)=\delta d(z)$, по индукции получим нужный результат.

\section{Список литературы}

[1] D. G. Birkhoff, W. J. Trjitzinsky, "Analytic theory of singular difference equations", Acta Math., 60:1 (1933), 1-89 doi 10.1007/BF02398269, MR 1555364, Zbl 59.0450.03.

[2] D. G. Birkhoff, "Formal theory of irregular linear difference equations", Acta Math., 54:1 (1930), 205-246 doi 10.1007/BF02547522, MR 1555307, Zbl 56.0402.01.

[3] D. G. Birkhoff, "General theory of linear difference equations", Trans. Amer. Math. Soc., 12:2 (1911), 243-284 doi 10.2307/1988577, MR 1500888, Zbl 42.0359.02. 